J. Lake Sci.(湖泊科学), 2020, 32(2): 528-538

DOI 10. $18307 / 2020.0221$

(c) 2020 by Journal of Lake Sciences

\title{
降雨动态系统响应曲线修正方法在黄土区域水沙模拟中的应用”
}

\author{
包为民 ${ }^{1}$, 顾雨薇 ${ }^{1}$, 司 伟 ${ }^{1 * *}$, 侯 露 $^{1}$, 卢金利 $^{2}$, 落全富 ${ }^{3}$ \\ $(1:$ 河海大学水文水资源学院,南京 210098) \\ (2:台州市水文站,台州 318001) \\ (3: 青山水库管理处,杭州 311305)
}

\begin{abstract}
摘 要: 采用水沙模型对流域水沙过程进行计算是目前分析和研究黄土地区水土流失、水沙锐减等问题的有效途径. 由 于降雨的时段均化和缺测、漏测、误测等问题, 导致水沙模型的重要输人项和动力因子一一降雨资料存在误差, 进而影响 水流和泥沙过程模拟精度. 因此, 本研究将降雨动态系统响应曲线的误差修正方法与概念性水沙模拟模型相结合以提高 水沙过程模拟精度. 此方法将水沙模型的水流模拟部分看作响应系统, 通过修正水沙模型的重要输人项一一面平均雨 量, 利用修正之后的面平均雨量系列, 通过模型重新计算以提高模型对产汇流和产汇沙过程的模拟精度. 通过理想案例 验证该方法可行性后, 选择黄土地区曹坪流域进行检验, 结果表明修正后的水流和泥沙过程模拟精度均有显著提高,平 均提高幅度分别为 $17.56 \%$ 和 $15.86 \%$.
\end{abstract}

关键词: 水沙模拟概念模型; 降雨误差修正;动态系统响应;曹坪流域

\section{Application of rainfall dynamic system response curve method for streamflow and sediment simulation in loess region*}

\author{
BAO Weimin ${ }^{1}$, GU Yuwei ${ }^{1}$, SI Wei ${ }^{1 * *}$, HOU Lu ${ }^{1}$, LU Jinli ${ }^{2} \&$ LUO Quanfu ${ }^{3}$ \\ (1: College of Hydrology and Water Resources, Hohai University, Nanjing 210098, P.R.China) \\ (2: Taizhou Hydrological Station, Taizhou 318001, P.R.China) \\ (3: Qingshan Reservoir Management Office, Hangzhou 311305, P.R.China)
}

Abstract: It is an effective way to use the conceptual model of water and sediment to calculate the water-sediment process in loess
region to analyze the current soil erosion and water-sediment reduction problems. There are errors in the rainfall data due to the tem-
poral homogenization, missing measurements and mismeasurements, which influence the accuracy of the important input variable
and dynamic factor of the model - rainfall, and then affect the accuracy of flow and sediment process simulation. Therefore, this
study combines the rainfall dynamic system response curve method with the conceptual model of water and sediment to improve the
accuracy of water and sediment process simulation. This method takes the water simulation part of the model as a response system to
update the important input variable-mean area rainfall. Then the runoff, sediment yield and concentration is recalculated using the
updated rainfall series to improve the accuracy of flow and sediment process simulation. After validating the feasibility of this meth-
od by the ideal case, an actual case is occurred in Caoping Basin in loess region. The results show that the method can both signifi-
cantly improve the accuracy of the water and sediment simulation, and the average increases are $17.56 \%$ and $15.86 \%$ respectively.
Keywords: Conceptual model of water and sediment simulation; rainfall error correction; dynamic system response; Caoping Basin

黄土地区泥沙问题是目前水文研究的重点问题之一. 研究黄河水沙变化、把握黄河水沙变化规律, 对于

* 2019-09-11 收稿; 2019-09-18 收修改稿.

国家重点研发计划专项( 2016YFC0402703)、中央级公益性科研院所基本科研业务费专项 (HKY-JBYW-2017-12)、 国家自然科学基金项目 (51709077)、博士后基金项目(2017M611679/1701019A) 和水利部黄河泥沙重点实验室开 放基金项目 (201804) 联合资助.

** 通信作者; E-mail: lindongsisi@163.com. 
进一步完善治黄方略、实施流域水资源配置与管理及重大水利工程布局, 意义重大 ${ }^{[1]}$. 目前泥沙模型的研究 取得了较为丰硕的成果, 主要包括经验相关模型和物理成因模型. 经验相关模型中, Wischmeier WH 等 ${ }^{[2]}$ 提

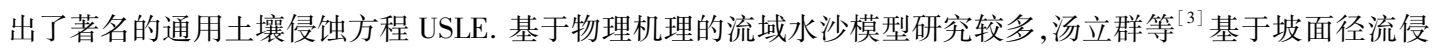
蚀量计算公式提出了流域产沙模型; WEPP、LISEM、EUROSEM 等模型 ${ }^{[4-6]}$ 基于土壤侵蚀过程的物理描述,模 拟了流域侵蚀过程, 在许多国家被成功推广应用. 已有的大多数流域产沙模型未能考虑不同流域尺度的泥 沙规律, 模型模拟精度以及使用范围受到了很大限制. 因此, 包为民 ${ }^{[7]}$ 基于流域自然地理特性, 在对黄土地 区产沙机制进行概化的基础上, 提出了结构和参数均具有物理意义的流域水沙模拟概念模型. 该模型经黄 土地区不同流域尺度的实际流域和前期大量的试验流域验证, 可以很好地模拟黄土高原地区流域的水沙 规律 $^{[8]}$.

水沙模拟中重要的动力条件是水流,因此水流模拟的精度直接影响流域产沙和汇沙过程,进而影响水 沙模型的模拟精度和效果 ${ }^{[9]}$. 水流模拟的主要技术手段是依赖于水文模型. 水文模型计算误差来源有很多, 其中包括重要输人项降雨资料、下垫面条件、流域初始状态值、模型本身误差等 ${ }^{[10]}$. 由于降雨是水文预报模 型计算的重要输人项, 因此降雨资料直接影响模型产流, 进而影响模型的模拟精度 ${ }^{[11]}$. 在水沙模型计算过 程中, 基于黄土区域自然地理特性, 流域面平均雨量大多采用 “以点带面” 的方式进行计算, 即利用雨量站点 的观测资料代表该雨量站所代表的单元面上的降雨, 这种方式不可避免的给流域降雨带来误差. 同时, 部分 降雨资料由于观测等因素存在时段均化现象, 伴有一定程度的缺测、漏测和误测问题, 降雨资料由插补展延 而来, 造成模型模拟误差. 因此需要对输人水沙模型进行计算的流域面平均雨量进行修正, 从而提高洪水和 泥沙的模拟精度. 国内外学者对降雨资料观测误差的分析与估计, 目前研究较多的是对雷达测雨资料误差 进行的修正, 然而以遥测系统为观测手段, 对流域尺度范围的降雨观测资料误差进行分析和研究则开展得 较少, 在国外几乎很少涉及 ${ }^{[12-15]}$. 为提高模型模拟精度, 司伟等 ${ }^{[16-17]}$ 首先提出了动态系统响应曲线修正方 法, 该方法是向误差源头追溯的动态反馈修正方法, 具有物理基础强和不损失预见期等优点 ${ }^{[18]}$. 将该方法 应用在新安江模型降雨误差估计中, 对流域面平均雨量进行修正以提高预报精度,取得了非常好的修正效 果 $^{[10,19-21]}$. 基于以上研究, 为了更精确模拟黄土区域水沙规律, 本文尝试将该方法应用在水沙模拟概念模型 中, 通过矫正降雨误差, 以提高水流和泥沙的模拟精度.

因此在水沙模拟概念模型计算中引人降雨动态系统响应曲线修正方法, 对流域面平均雨量进行修正, 建立面平均雨量和流域流量之间的响应关系. 降雨和产汇流过程是流域产沙汇沙过程的动力来源, 水流是 泥沙运移和输送的载体, 水流模拟精度与流域出口断面的输沙率过程息息相关. 鉴于此, 本研究尝试通过对 面平均雨量进行修正, 将修正后面平均雨量系列输人模型进行计算重新得到新的流量和输沙率过程, 以达 到提高水沙模型模拟精度的目的.

\section{1 水沙模拟概念模型介绍}

水沙模拟概念模型是包为民教授在 20 世纪末研究黄土地区流域泥沙提出的概念性模型, 并被用于模 拟蛇家沟和水旺沟流域等 20 多个小流域以及皇甫和河口镇-龙门镇区间等中大流域的水沙变化, 取得了较 好的模拟效果. 模型结构简单, 物理概念清晰, 适用于黄土区域干旱半干旱地区, 以满足水土保持措施效益 分析和黄河中游水沙变化原因分析等问题研究的大尺度、长系列模拟的需要, 为人类活动和气候变化对流 域水沙变化的影响提供基础数据 ${ }^{[22]}$.

该模型根据水沙在流域上产生、运动各个环节机制的差异, 分为水流模拟和泥沙模拟两大部分 ${ }^{[23]}$. 水 流模拟分为产流、坡面汇流和沟道汇流 3 部分; 泥沙模拟分为面蚀产沙、沟蚀产沙、坡面汇沙和沟道汇沙 4 部 分. 产流机制采用垂向混合产流模式,坡面汇流采用线性水库法, 沟道汇流采用马斯京根河道演算法; 面蚀 产沙采用土壤抗侵蚀能力分布曲线, 沟蚀产沙采用拜格诺河道水流悬移质泥沙公式, 坡面汇沙和沟道汇沙 采用泥沙汇集概念模拟公式.

模型输人为降雨 $P$ 和蒸发 $E$ 资料, 主要输出为流域出口断面的流量过程和泥沙过程. 模型主要结构和 参数如图 1 和表 1 所示. 


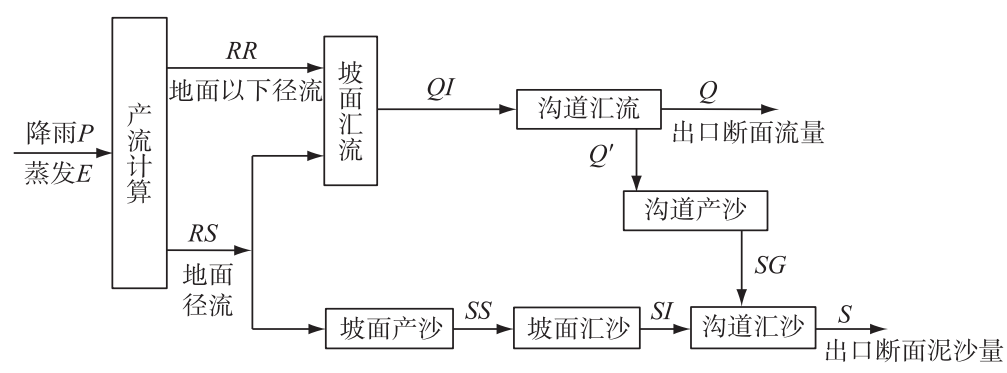

图 1 水沙模拟概念模型结构图

Fig. 1 Structural chart of conceptual model of water and sediment simulation

表 1 水沙模拟概念模型参数

Tab.1 Parameters of conceptual model of water and sediment simulation

\begin{tabular}{|c|c|c|c|c|}
\hline 种类 & 参数符号 & 参数意义 & 敏感程度 & 取值范围 \\
\hline \multirow[t]{4}{*}{ 产流 } & $W M / \mathrm{mm}$ & 流域平均土壤蓄水含量 & 敏感 & $180 \sim 350$ \\
\hline & $F C /(\mathrm{mm} / \mathrm{min})$ & 流域平均稳定下渗率 & 敏感 & $0.1 \sim 10$ \\
\hline & $B F$ & 流域下渗率分布曲线指数 & 不敏感 & $0.1 \sim 2$ \\
\hline & $K F$ & 土壤对下渗率影响系数 & 不敏感 & $0.1 \sim 2$ \\
\hline \multirow[t]{7}{*}{ 汇流 } & $B$ & 土壤蓄水容量分布曲线指数 & 敏感 & $0.1 \sim 1$ \\
\hline & $K G$ & 自由水箱地下水出流系数 & 敏感 & $0.1 \sim 1$ \\
\hline & $C S$ & 地面径流线性水库消退系数 & 敏感 & $0.1 \sim 0.65$ \\
\hline & $C I$ & 壤中流线性水库消退系数 & 敏感 & $0.65 \sim 0.95$ \\
\hline & $C G$ & 地下径流线性水库消退系数 & 敏感 & $0.95 \sim 0.9999$ \\
\hline & $K E / \min$ & 马斯京根法河段传播时间 & 敏感 & $0.5 \sim 2$ \\
\hline & $X E$ & 马斯京根法流量比重系数 & 敏感 & $0.05 \sim 0.55$ \\
\hline \multirow[t]{5}{*}{ 产沙 } & $A C M /\left(\mathrm{kg} / \mathrm{m}^{3}\right)$ & 坡面水力侵蚀能力系数 & 敏感 & $0.001 \sim 1$ \\
\hline & $R E M M / \mathrm{kg}$ & 坡面最大抗侵蚀能力 & 不敏感 & $>0$ \\
\hline & $B S$ & 抗侵蚀能力分布曲线指数 & 不敏感 & $0.1 \sim 5$ \\
\hline & $C M /\left(\mathrm{kg} / \mathrm{m}^{3}\right)$ & 断面最大含沙量 & 不敏感 & $5 \sim 2000$ \\
\hline & $\operatorname{CSS} /\left(\mathrm{kg} / \mathrm{m}^{3}\right)$ & 坡面毛沟侵蚀参数 & 敏感 & $0.1 \sim 250$ \\
\hline \multirow[t]{4}{*}{ 汇沙 } & $K X D$ & 非线性冲刷系数 & 敏感 & $0.1 \sim 0.999$ \\
\hline & $K S / \min$ & 河段输沙平均传播时间 & 敏感 & $0.5 \sim 3$ \\
\hline & $X S$ & 河段输沙权重系数 & 敏感 & $0.1 \sim 0.55$ \\
\hline & $\zeta$ & 冲淤系数 & 敏感 & $0.01 \sim 1$ \\
\hline
\end{tabular}

\section{2 降雨动态系统响应方法介绍}

动态系统响应曲线修正方法是用于水文预报模型中的误差修正技术, 以模型输人或某中间变量与输出 之间的对应关系建立响应曲线. 由于降雨是模型的重要输人项, 降雨误差会在模型计算中进行传递 ${ }^{[24]}$. 因 此通过修正流域面平均雨量, 提高模型的模拟精度.

本文将该方法用于水沙模拟概念模型中, 将模型的水流模拟部分概化为一个系统 (图 2), 建立动态系统 响应曲线函数 ${ }^{[17]}$ :

$$
Q(t)=f[X(t), \theta, t]
$$

式中, $Q=\left[Q_{1}, Q_{2}, Q_{3} \cdots \cdots Q_{n}\right]^{\mathrm{T}}$ 为流域出口断面流量过程 $\left(\mathrm{m}^{3} / \mathrm{s}\right) ; S=\left[S_{1}, S_{2}, S_{3} \cdots \cdots S_{n}\right]^{\mathrm{T}}$ 为流域出口断 面输沙率过程 $(\mathrm{kg} / \mathrm{s}) ; P=\left[P_{1}, P_{2}, P_{3} \cdots \cdots P_{n}\right]^{\mathrm{T}}$ 为流域降雨系列 $(\mathrm{mm}) ; E=\left[e_{1}, e_{2}, e_{3} \cdots \cdots e_{n}\right]^{\mathrm{T}}$ 为蒸发 $(\mathrm{mm}) ; X(t)$ 为模型的输人变量和中间变量, $X_{0}$ 为模型变量初始状态值; $\theta$ 为模型参数, 在一定时期内不随时 
间变化; $t$ 为时间, $n$ 为系列长度.

在本文中,只考虑降雨对水沙模型流量的响应过程,因此降雨 动态系统响应曲线函数简化为:

$$
Q(P)=f(P)
$$

所谓降雨动态系统响应曲线,即对某时段的降雨 $P_{i}$ 增加一个 单位值,将新的降雨系列输人模型进行计算得到的流量与原始计 算流量之间的差值.

把降雨输人作为自变量对流量求全微分, 并将观测流量系列 代人式中,则其矩阵表达式为:

$$
Q(P) \approx Q C\left(P_{0}\right)+U \Delta P+W
$$

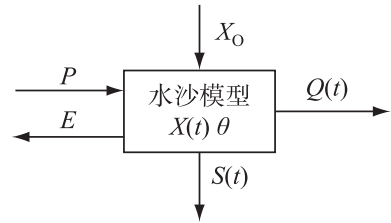

图 2 水沙模型概化系统

Fig. 2 Generalization system of conceptual model of water and sediment simulation

式中, $\Delta P$ 是需求解的降雨系列误差 $(\mathrm{mm}) ; W$ 是经过实时修正后的预报系统残差 $\left(\mathrm{m}^{3} / \mathrm{s}\right) ; U$ 为系统响应 矩阵.

$U$ 矩阵如下:

$$
U=\left[\begin{array}{ccc}
\frac{\partial Q_{1}(P)}{\partial P_{1}} & \cdots & \frac{\partial Q_{1}(P)}{\partial P_{m}} \\
\frac{\partial Q_{2}(P)}{\partial P_{1}} & \cdots & \frac{\partial Q_{2}(P)}{\partial P_{m}} \\
\cdots & \cdots & \cdots \\
\frac{\partial Q_{n}(P)}{\partial P_{1}} & \cdots & \frac{\partial Q_{n}(P)}{\partial P_{m}}
\end{array}\right]
$$

计算降雨动态系统响应曲线的步骤如下:

（1）假设流域有降雨系列 $P_{0}$, 实测流量为 $Q_{0}, P_{0}$ 输人模型计算得到流域原始计算流量系列为 $Q_{\mathrm{C}}$.

(2) 流域各站点降雨系列 $P_{0}$ 中第 $i$ 个时段 $P_{i}$, 在其余时段各站点降雨均不变的情况下,增加一个单位 值, 得到新的降雨系列 $P_{\mathrm{C} i}$.

(3) 将新的降雨系列 $P_{C i}$ 输人模型进行计算,得到新的计算流量过程 $Q_{C i}$.

(4) 将新的流量过程 $Q_{\mathrm{C} i}$ 与原始计算流量 $Q_{\mathrm{C}}$ 相减, 其差值为 $P_{i}$ 的系统响应曲线, 属于 $U$ 矩阵中的第 $i$ 列.

(5) 依次计算 $U$ 矩阵中的每一列, 最终建立降雨系列 $P_{0}$ 的动态系统响应曲线. 运用最小二乘法,计算得 到降雨的修正误差值:

(6) 修正后的降雨系列:

$$
\Delta P=\left(U^{\mathrm{T}} U\right)^{-1} U^{\mathrm{T}}\left(Q_{0}-Q_{\mathrm{C}}\right)
$$

$$
P_{\mathrm{U}}=P_{0}+\Delta P
$$

输人模型进行计算, 新的流域出口断面流量和输沙率过程即该流域流量和输沙率的修正值.

\section{3 应用检验}

检验方法修正效果,具体采用以下 4 个指标来衡量修正效果:

(1) 径流深相对误差 $\Delta R$, 本文取误差绝对值:

$$
\Delta R=\left|\frac{R_{\mathrm{C}}-R_{0}}{R_{0}} \times 100 \%\right|
$$

(2) 产沙量相对误差 $\Delta S$, 本文取误差绝对值:

$$
\Delta S=\left|\frac{S_{\mathrm{C}}-S_{0}}{S_{0}} \times 100 \%\right|
$$

(3) 纳什效率系数 NS : 


$$
N S=1-\frac{\sum_{i}^{n}\left[y_{\mathrm{C} i}-y_{\mathrm{O} i}\right]^{2}}{\sum_{i}^{n}\left[y_{\mathrm{O} i}-\overline{y_{\mathrm{O}}}\right]^{2}}
$$

(4) 纳什效率系数提高幅度 INS :

$$
I N S=\frac{N S_{\mathrm{U}}-N S_{0}}{N S_{0}} \times 100 \%
$$

式中, $R_{\mathrm{C}}$ 为径流深预报值 $(\mathrm{mm}) ; R_{0}$ 为径流深实测值 $(\mathrm{mm}) ; S_{\mathrm{C}}$ 为产沙量预报值 $(\mathrm{t}) ; S_{\mathrm{O}}$ 为产沙量实测值 $(\mathrm{t}) ; y_{\mathrm{C} i}$ 为观测变量第 $i$ 个时段预报值; $y_{\mathrm{O} i}$ 为观测变量第 $i$ 个时段实测值; $\overline{y_{0}}$ 为观测变量实测值的均值; $n$ 为 资料系列长度; $N S_{0}$ 为修正前纳什效率系数; $N S_{\mathrm{U}}$ 为修正后纳什效率系数.

\section{1 理想案例检验}

在理想案例中, 假设模型的输人、输出、参数和初始状态值已知. 假设一个降雨系列 $P_{0}$, 将其输人水沙模 型计算得到的流量和输沙率 $Q_{0}$ 和 $S_{0}$, 作为理想案例的实测系列. 随机给定一组服从零均值分布的降雨误 差值 $\Delta P$, 误差值不超过降雨系列的 $\pm 80 \%$, 将加上误差值的新的降雨系列 $P_{\mathrm{C}}$ 输人模型计算得到计算流量 $Q_{\mathrm{C}}$ 和计算输沙率 $S_{\mathrm{C}}$. 其中,

$$
P_{\mathrm{C}}=P_{\mathrm{O}}+\Delta P
$$

对 $P_{\mathrm{C}}$ 系列降雨进行动态系统响应修正, 修正得到降雨误差值为 $-\Delta P^{\prime}$, 修正的降雨系列为:

$$
P_{\mathrm{U}}=P_{\mathrm{C}}-\Delta P^{\prime}=P_{0}+\Delta P-\Delta P^{\prime}
$$

$P_{\mathrm{U}}$ 输人模型计算得到修正后流量 $Q_{\mathrm{U}}$ 和输沙率 $S_{\mathrm{U}}$. 分别将修正降雨误差值 $-\Delta P^{\prime}$ 与给定降雨误差值 $\Delta P$ 进行对比, 比较两者相关性, 检验该方法是否能够精确反演降雨误差; 分别将修正后流量、输沙率和计算流 量、输沙率与实测流量、输沙率进行对比分析,检验修正后的模型模拟精度是否得到提高.

在本研究中,理想案例降雨系列采用曹坪流域 1962 年 7 月 23 日场次洪水降雨资料,流域面积 $187 \mathrm{~km}^{2}$, 有 30 个雨量站, 洪水汇流演算马斯京根演算法河段数为 10 . 给定随机降雨误差值建立理想案例, 表 2 为理 想案例的径流深和产沙量修正情况, 图 3 为理想案例给定降雨误差和修正降雨误差值对比图, 图 4 为理想案

\begin{tabular}{|c|c|c|c|c|c|c|c|}
\hline \multirow{2}{*}{ 水流 } & \multicolumn{4}{|c|}{ 修正前 } & \multicolumn{3}{|c|}{ 修正后 } \\
\hline & $R_{0}{ }^{1)} / \mathrm{mm}$ & $R_{\mathrm{C}}^{2)} / \mathrm{mm}$ & $\Delta R_{1}^{3)} / \%$ & $N S_{0}$ & $R_{\mathrm{U}}^{4)} / \mathrm{mm}$ & $\Delta R_{2}{ }^{5)} / \%$ & $N S_{\mathrm{U}}$ \\
\hline & 3.74 & 3.43 & 8.29 & 0.989 & 3.64 & 2.67 & 0.997 \\
\hline \multirow{3}{*}{ 泥沙 } & \multicolumn{4}{|c|}{ 修正前 } & \multicolumn{3}{|c|}{ 修正后 } \\
\hline & $S_{0}{ }^{6)} / \mathrm{t}$ & $S_{\mathrm{C}}{ }^{7)} / \mathrm{t}$ & $\Delta S_{1}^{8)} / \%$ & $N S_{0}$ & $S_{\mathrm{U}}{ }^{9)} / \mathrm{t}$ & $\Delta S_{2}{ }^{10)} / \%$ & $N S_{\mathrm{U}}$ \\
\hline & 3103 & 3319 & 6.96 & 0.990 & 3251 & 4.77 & 0.996 \\
\hline
\end{tabular}
例降雨数据的修正效果, 图 5 为理想案例水流和泥沙修正效果.

表 2 理想案例流量和输沙率修正效果

Tab.2 Correction effect of flow and sediment transport rate of ideal case

1) $R_{0}$ 为实测径流深;2) $R_{\mathrm{C}}$ 为模型原始预报径流深;3) $\Delta R_{1}$ 为模型原始预报径流深与实测径流深之间相对误差;4) $R_{\mathrm{U}}$ 为模 型修正后径流深;5) $\Delta R_{2}$ 为模型修正径流深与实测径流深之间相对误差;6) $S_{0}$ 为实测产沙量 ; 7) $S_{\mathrm{C}}$ 为模型原始计算产沙 量;8) $\Delta S_{1}$ 为模型原始计算产沙量与实测产沙量之间相对误差;9) $S_{\mathrm{U}}$ 为模型修正后产沙量; 10) $\Delta S_{2}$ 为模型修正产沙量与 实测产沙量之间相对误差.

从表 2 可以看出, 应用降雨动态系统响应曲线后, 理想案例水流部分径流深误差由 $8.29 \%$ 降低到 $2.67 \%$, 纳什效率系数由 0.989 提高到 0.997 ; 泥沙部分产沙量相对误差由 $6.96 \%$ 降低到 $4.77 \%$, 纳什效率系 数由 0.990 提高到 0.996. 从图 3 可以看出, 给定降雨误差与修正得到的降雨误差之间 $N S$ 系数为 0.821 , 相关 系数为 0.908 , 二者非常接近. 从图 4 可以看出, 相比较有误差的降雨系列 $P_{\mathrm{C}}$, 修正后的降雨系列 $P_{\mathrm{U}}$ 更接近 于降雨基准数据 $P_{0}$ 系列, 这说明本文中所采用的降雨误差估计方法能够准确地估计出给定的降雨误差, 精 
确反演降雨误差. 从图 5 可以看出, 修正后的流量和输沙率更接近流量真值和输沙率真值, 流量和输沙率拟 合过程都有所改善. 这说明降雨动态系统响应曲线修正方法能够同时提高水沙模型的水流和泥沙模拟精 度, 可以进一步用于实际流域的应用检验.

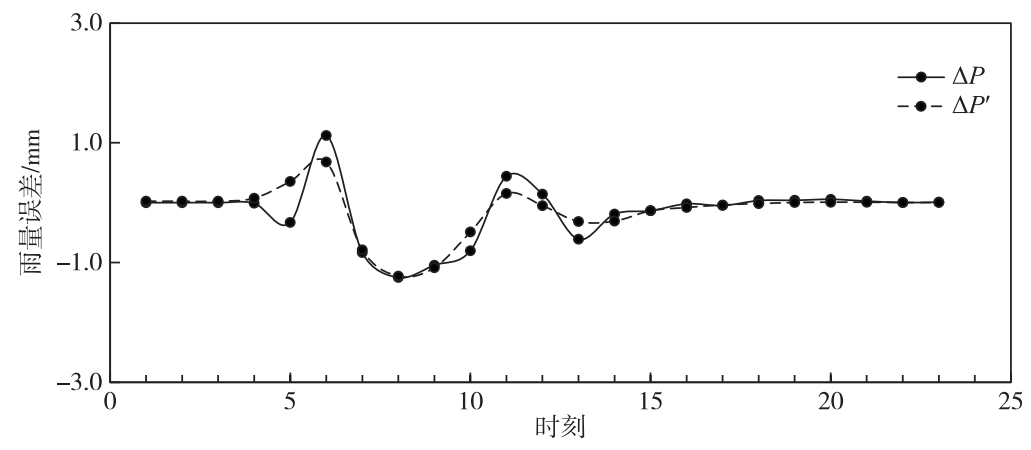

图 3 理想案例给定雨量误差 $\Delta P$ 及修正雨量误差 $\Delta P^{\prime}$ 值 (图中 1 个时刻为模型计算时间尺度 $0.5 \mathrm{~h}$ )

Fig.3 Given rainfall error $\Delta P$ and corrected rainfall error $\Delta P^{\prime}$ of ideal case

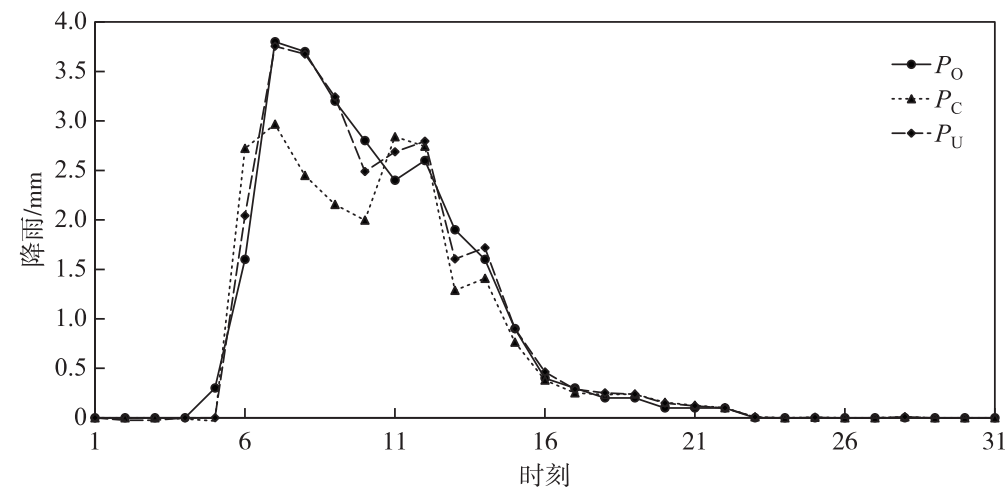

图 4 理想案例修正前后降雨值(图中 1 个时刻为模型计算时间尺度 $0.5 \mathrm{~h}$ )

Fig.4 Rainfall values before and after revision of ideal case
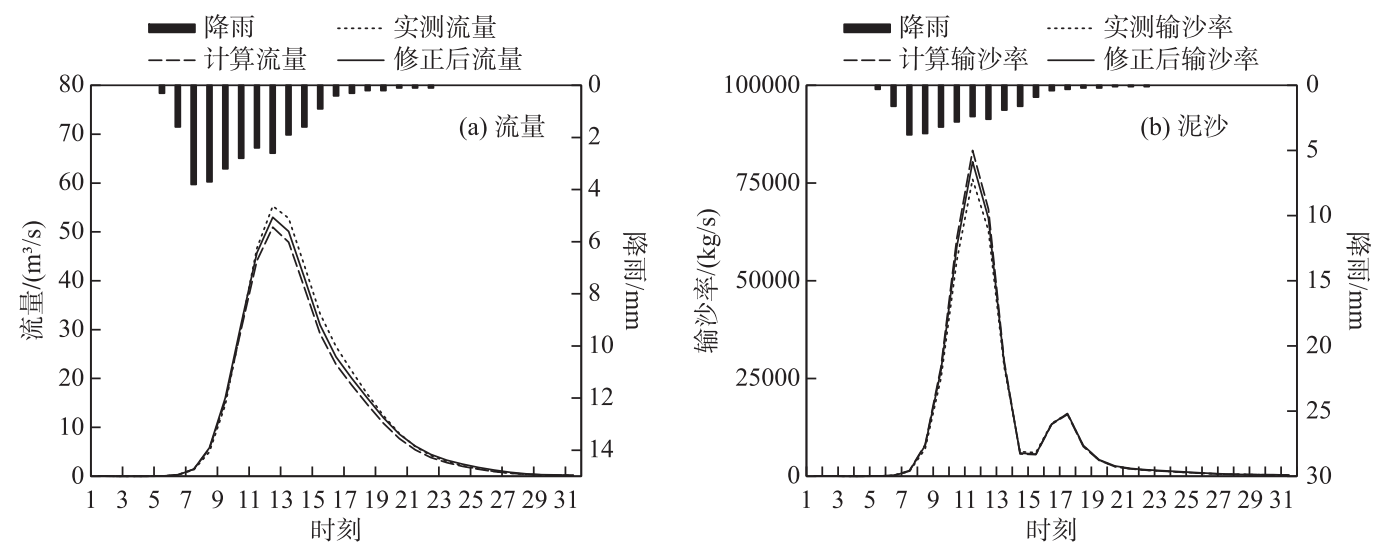

图 5 理想案例修正效果 (图中 1 个时刻为模型计算时间尺度 $0.5 \mathrm{~h}$ )

Fig.5 Correction effect of ideal case 


\section{2 实际流域应用}

本文选取黄土地区曹坪流域作为实验流域. 曹坪流域位于黄河大理河流域中下游地区, 流域大部分为 黄土丘陵沟壑区域, 植被稀疏, 水土流失严重. 是典型的大陆性季风气候区, 夏季炎热潮湿多暴雨, 冬季寒冷 干燥. 降雨年内变化大, 降雨多集中在 7-9 月. 流域多年平均降雨量 $447 \mathrm{~mm}$, 多年平均气温 $7.8 \sim 9.6^{\circ} \mathrm{C}$, 流 域泥沙侵蚀模数为 2.2 万 $\mathrm{t} /\left(\mathrm{km}^{2} \cdot \mathrm{a}\right)$.

研究流域曹坪 (岔巴沟) 为干旱半干旱气候, 产流模式符合混合产流模式, 可以运用水沙概念模拟模型 进行计算. 用于计算修正的资料系列为 1961-1989 年的 13 场洪水资料,模型计算时间尺度为 $0.5 \mathrm{~h}$, 流域水 系及站网布设见图 6.

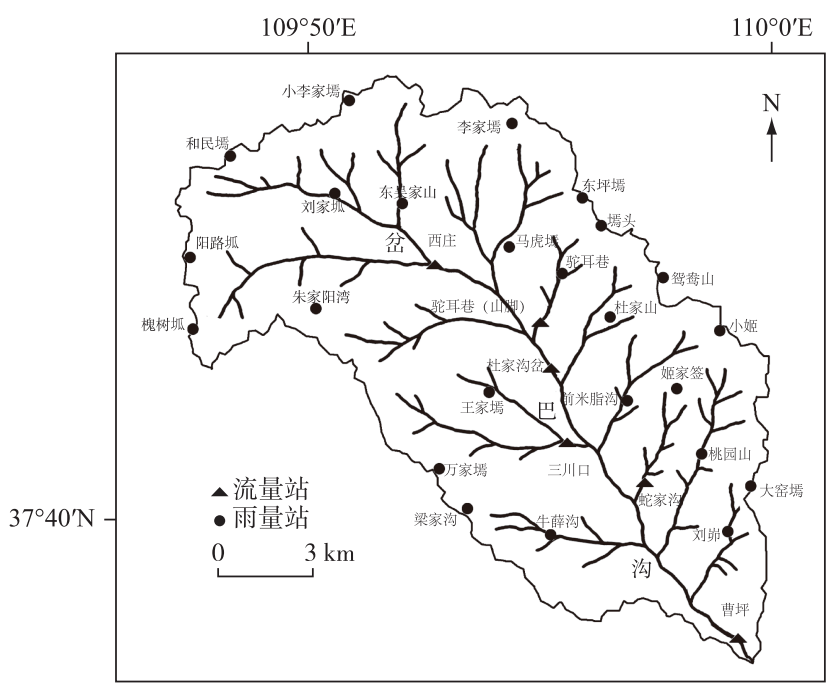

图 6 曹坪流域水系及站网布设

Fig.6 Layout of water system and station network in Caoping Basin

水沙模型经函数曲面参数率定方法 ${ }^{[25]}$ 进行参数率定后, 对降雨进行动态系统响应修正, 检验模型的修 正效果. 模型参数率定情况见表 3. 13 场洪水水流和泥沙修正结果分别见表 4 和表 5.

表 3 水沙模型曹坪流域参数率定

Tab.3 Calibration of parameters of Caoping Basin for conceptual model of water and sediment simulation

\begin{tabular}{cccc}
\hline 水流参数 & 率定值 & 泥沙参数 & 率定值 \\
\hline$W M / \mathrm{mm}$ & 348.95 & $A C M /\left(\mathrm{kg} / \mathrm{m}^{3}\right)$ & 0.33 \\
$F C /(\mathrm{mm} / \mathrm{min})$ & 9.97 & $R E M M / \mathrm{kg}$ & 1000 \\
$B F$ & 0.11 & $B S$ & 1 \\
$K F$ & 0.84 & $C M /\left(\mathrm{kg} / \mathrm{m}^{3}\right)$ & 1575.63 \\
$B$ & 0.22 & $C S S /\left(\mathrm{kg} / \mathrm{m}^{3}\right)$ & 1.3 \\
$K G$ & 0.25 & $K S / \mathrm{min}$ & 0.62 \\
$C S$ & 0.10 & $X S$ & 0.2 \\
$C I$ & 0.65 & $\zeta$ & 0.03 \\
$C G$ & 0.995 & & \\
$X E$ & 0.5 & & \\
\hline
\end{tabular}


表 4 曹坪流域水流修正效果

Tab.4 Correction effect of the water simulation part of Caoping Basin

\begin{tabular}{|c|c|c|c|c|c|c|c|c|}
\hline \multirow{2}{*}{ 洪号 } & \multicolumn{4}{|c|}{ 修正前 } & \multicolumn{4}{|c|}{ 修正后 } \\
\hline & $R_{0} / \mathrm{mm}$ & $R_{\mathrm{C}} / \mathrm{mm}$ & $\Delta R_{1} / \%$ & $N S_{0}$ & $R_{\mathrm{U}} / \mathrm{mm}$ & $\Delta R_{2} / \%$ & $N S_{\mathrm{U}}$ & $I N S / \%$ \\
\hline 1 & 1.31 & 1.32 & 0.76 & 0.703 & 1.3 & 0.76 & 0.852 & 21.19 \\
\hline 2 & 3.46 & 3.43 & 0.87 & 0.646 & 3.48 & 0.58 & 0.751 & 16.25 \\
\hline 3 & 3.47 & 3.46 & 0.29 & 0.643 & 3.47 & 0.00 & 0.745 & 15.86 \\
\hline 4 & 0.93 & 0.95 & 2.15 & 0.604 & 0.95 & 2.15 & 0.801 & 32.62 \\
\hline 5 & 1.45 & 1.23 & 15.17 & 0.767 & 1.44 & 0.69 & 0.812 & 5.87 \\
\hline 6 & 1.47 & 1.23 & 16.33 & 0.761 & 1.37 & 6.80 & 0.866 & 13.80 \\
\hline 7 & 2.99 & 2.97 & 0.67 & 0.788 & 2.99 & 0.00 & 0.906 & 14.97 \\
\hline 8 & 0.75 & 0.74 & 1.33 & 0.688 & 0.74 & 1.33 & 0.812 & 18.02 \\
\hline 9 & 3.58 & 3.55 & 0.84 & 0.699 & 3.57 & 0.28 & 0.725 & 3.72 \\
\hline 10 & 10.74 & 10.67 & 0.65 & 0.700 & 10.75 & 0.09 & 0.890 & 27.14 \\
\hline 11 & 9.38 & 8.99 & 4.16 & 0.690 & 9.15 & 2.45 & 0.814 & 17.97 \\
\hline 12 & 0.94 & 0.95 & 1.06 & 0.607 & 0.94 & 0.00 & 0.704 & 15.98 \\
\hline 13 & 1.41 & 1.34 & 4.96 & 0.657 & 1.46 & 3.55 & 0.851 & 29.53 \\
\hline 平均 & 3.22 & 3.14 & 2.48 & 0.689 & 3.20 & 0.62 & 0.810 & 17.56 \\
\hline
\end{tabular}

表 5 曹坪流域泥沙修正效果

Tab.5 Correction effect of the sediment simulation part of Caoping Basin

\begin{tabular}{|c|c|c|c|c|c|c|c|c|}
\hline \multirow{2}{*}{ 洪号 } & \multicolumn{4}{|c|}{ 修正前 } & \multicolumn{4}{|c|}{ 修正后 } \\
\hline & $S_{0} / \mathrm{t}$ & $S_{\mathrm{C}} / \mathrm{t}$ & $\Delta S_{1} / \%$ & $N S_{0}$ & $S_{\mathrm{U}} / \mathrm{t}$ & $\Delta S_{2} / \%$ & $N S_{\mathrm{U}}$ & INS $/ \%$ \\
\hline 1 & 582 & 648 & 11.34 & 0.600 & 636 & 9.28 & 0.740 & 23.33 \\
\hline 2 & 2995 & 2461 & 17.83 & 0.632 & 2475 & 17.36 & 0.734 & 16.14 \\
\hline 3 & 2254 & 1653 & 26.66 & 0.628 & 1883 & 16.46 & 0.759 & 20.86 \\
\hline 4 & 528 & 466 & 11.74 & 0.605 & 473 & 10.42 & 0.680 & 12.40 \\
\hline 5 & 1044 & 816 & 21.84 & 0.650 & 817 & 21.74 & 0.713 & 9.69 \\
\hline 6 & 1035 & 852 & 17.68 & 0.614 & 870 & 15.94 & 0.712 & 15.96 \\
\hline 7 & 1765 & 1529 & 13.37 & 0.679 & 1532 & 13.20 & 0.815 & 20.03 \\
\hline 8 & 426 & 450 & 5.63 & 0.688 & 429 & 0.70 & 0.720 & 4.65 \\
\hline 9 & 2243 & 2760 & 23.05 & 0.622 & 2615 & 16.58 & 0.673 & 8.20 \\
\hline 10 & 4669 & 4939 & 5.78 & 0.710 & 4870 & 4.30 & 0.834 & 17.46 \\
\hline 11 & 5662 & 7166 & 26.56 & 0.634 & 6980 & 23.28 & 0.809 & 27.60 \\
\hline 12 & 443 & 473 & 6.77 & 0.636 & 450 & 1.58 & 0.736 & 15.72 \\
\hline 13 & 748 & 587 & 21.52 & 0.656 & 673 & 10.03 & 0.760 & 15.85 \\
\hline 平均 & 1876 & 1908 & 1.71 & 0.643 & 1900 & 1.28 & 0.745 & 15.86 \\
\hline
\end{tabular}

从表 4 洪水修正效果来看, 应用降雨动态系统响应曲线后, 修正后的径流深相对误差与修正前相比都 有所减小,纳什效率系数均有所提高. 平均径流深相对误差由 $2.48 \%$ 降低到 $0.62 \%$, 纳什效率系数由 0.689 提高到 0.810 ,纳什效率系数提高幅度为 $17.56 \%$. 从表 5 泥沙修正效果来看,产沙量经修正后相对误差全部 降低, 纳什效率系数全部提高. 平均产沙量相对误差从 $1.71 \%$ 降低到 $1.28 \%$, 纳什效率系数从 0.643 提高到 0.745 , 纳什效率系数提高幅度为 $15.86 \%$. 从图 7 洪水的纳什效率系数修正效果来看,修正后的纳什效率系 数均比修正前有显著提升. 选取第 8 场次洪水的流量和输沙率修正结果查看修正效果, 从图 8 可以看出, 洪 水修正后的流量和输沙率更接近流量真值和输沙率真值, 流量和输沙率拟合过程也有所改善. 这说明降雨 动态系统响应曲线修正方法应用于水沙模型后, 能够减小模型径流深和产沙量的相对误差, 提高纳什效率 

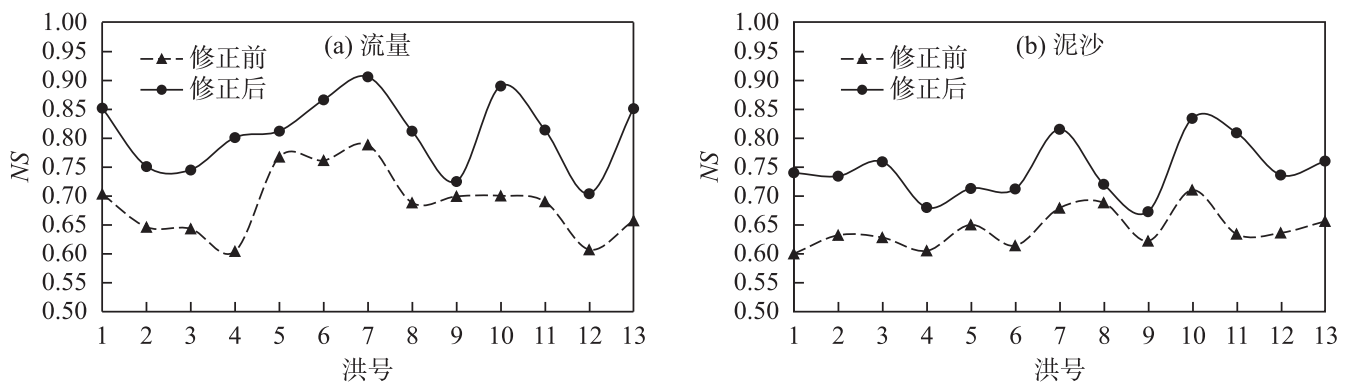

图 7 曹坪流域 13 场洪水纳什效率系数修正前后对比

Fig. 7 Contrast of NS coefficient before and after correction of 13 floods in Caoping Basin
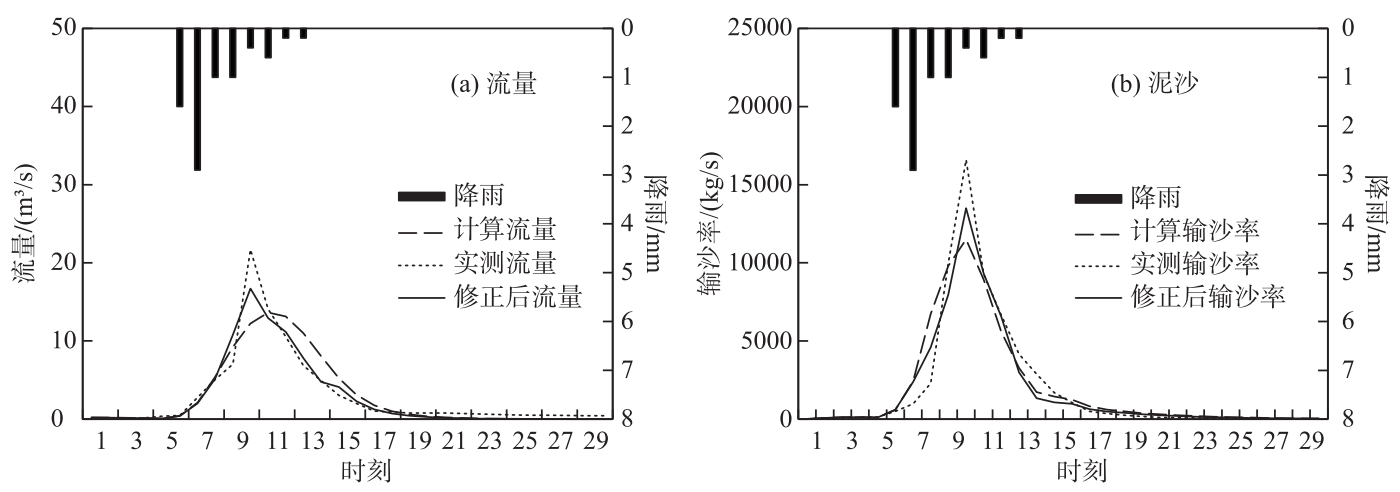

图 8 第 8 场次洪水修正效果 (图中 1 个时刻为模型计算时间尺度 $0.5 \mathrm{~h}$ )

Fig. 8 Correction effect of flood 8

系数,最终提高模型的模拟精度.

在现今应用的黄河流域土壤侵蚀产沙模型中, 流域的复杂性和资料的制约, 造成模型模拟结果精度欠 佳的不足 ${ }^{[26]}$. 流域面平均雨量的误差, 影响了模型的模拟精度, 使模型初始模拟效果没有想象中理想. 用于 降雨动态系统响应修正的 13 场洪水降雨大多分为两种情况:一是由于原始降雨资料存在降雨时段均化、缺 测、漏测等问题, 部分降雨数据由插补展延而来导致降雨误差. 这种类型降雨输人模型进行计算后, 相比实 际流量和输沙率过程, 计算洪水流量和输沙率过程相对呈现 “矮胖型”, 洪水缓涨缓落, 洪峰、沙峰偏低, 模型 模拟精度不高. 二是由于原始资料存在误测等问题, 某些时刻降雨数据明显偏大或者偏小, 造成降雨数据误 差. 这部分降雨数据输入模型后也会造成计算误差, 使计算流量和输沙率过程与实际过程不相符合, 模型模 拟精度较低. 降雨是模型计算的重要输人项, 也是模型误差的重要来源之一. 因此, 在使用水沙模型模拟黄 土区域水沙时, 对于有误差的降雨资料, 尤其针对以上两种降雨分布情况的洪水场次, 可以应用降雨动态系 统响应曲线修正方法,通过修正面平均雨量, 以提高模型模拟精度.

\section{4 结论}

水沙模拟概念模型物理概念清楚, 实用性强, 适用于黄土区域干旱半干旱地区. 应用于黄土地区流域, 合理地模拟了流域水沙变化规律. 降雨是水沙模型的重要输人项, 降雨误差能够直接造成模型误差, 从而影 响模型的模拟精度. 因此, 本文将降雨动态系统响应曲线修正方法与水沙模型相结合, 通过修正面平均雨 量, 将修正后降雨输人模型进行计算重新得到新的流量和输沙率过程, 以达到减小模型误差的目的. 结果表 明, 在对降雨进行修正后, 水沙模型水流径流深更接近实际值, 流量过程纳什效率系数提高; 产沙量误差降 低, 输沙率过程纳什效率系数提高. 说明水沙模型应用降雨动态系统响应曲线修正方法后, 能够提高模型的 
模拟精度,最终水流和泥沙模拟平均提高幅度分别是 $17.56 \%$ 和 $15.86 \%$,其修正效果是显著的.

降雨动态系统响应曲线修正方法能够很好地提高水沙模型的模拟精度,但是由于水文系统毕竟是非线 性系统, 影响因素比较复杂, 降雨误差因素并非模型误差的唯一来源. 考虑到本文仅修正面平均雨量一个变 量, 因此, 对于如何细化降雨误差, 以及综合考虑降雨和其他影响因素之间量化关系有待进一步研究. 根据 流域降雨分布情况, 选择降雨动态系统响应曲线修正方法应用于水沙模型模拟中, 可以为研究黄河水沙变 化规律提供一定的技术指导和理论支撑.

\section{5 参考文献}

[ 1 ] Yao WY, Jiao P. Water and sediment variations and research prospect in the Yellow River. Soil and Water Conservation in China , 2016, (9) : 55-63, 93. [姚文艺, 焦鹏. 黄河水沙变化及研究展望. 中国水土保持, 2016, (9) : 55-63, 93.]

[ 2 ] Wischmeier WH, Smith DD. Predicting rainfall-erosion losses from cropland east of the Rocky Mountain. Agriculture Handbook, 1965, 282: 47.

[ 3 ] Tang LQ. Study on sediment yield model of basin. Advances in Water Science, 1996, (1): 47-53. [汤立群. 流域产沙模 型的研究. 水科学进展, 1996, (1): 47-53.]

[ 4 ] Laflen JM, Lane LJ, Foster GR. WEPP: A new generation of erosion prediction technology. Journal of Soil \& Water Conservation, 1991, 46(1): 34-38.

[ 5 ] De Roo APJ, Jetten VG. Calibrating and validating the LISEM model for two data sets from the Netherlands and South Africa. Catena, 1999, 37(3/4): 477-493.

[ 6 ] Morgan RPC, Quinton JN, Smith RE et al. The European soil erosion model (EUROSEM) : a dynamic approach for predicting sediment transport from fields and small catchments. Earth Surface Processes and Landforms, 1998, (23): 527-544.

[ 7 ] Bao WM. A conceptual modelling study for small basin sediment yields in Loess Region. Advances in Water Science, 1993, (1) : 44-50. [包为民. 黄土地区小流域产沙概念性模拟研究. 水科学进展, 1993, (1) : 44-50.]

[ 8 ] Bao WM, Hou L, Shen DD et al. Application of flow-sedimentation coupled model in Dali River Basin of Loess Plateau. $J$ Lake Sci, 2019, 31(4) : 1120-1131. DOI: 10.18307/2019.0407. [包为民, 侯露, 沈丹丹等. 黄土高原大理河流域水 沙耦合模型应用研究. 湖泊科学, 2019, 31(4): 1120-1131.]

[ 9 ] Bao WM ed. Conceptual model of water and sediment simulation and application in Loess Region. Nanjing: Press of Hohai University, 1995: 170. [包为民. 黄土地区流域水沙模型模拟概念模型与应用. 南京: 河海大学出版社, 1995: 170.]

[10] Si W, Bao WM, Qu SM. Runoff error correction in real-time flood forecasting based on dynamic system response curve. Advances in Water Science, 2013, 24(4) : 497-503. [司伟, 包为民, 翟思敏. 洪水预报产流误差的动态系统响应曲线 修正方法. 水科学进展, 2013, 24(4): 497-503.]

[11] Si W, Yu HH, Bao WM et al. Area-mean rainfall updating by system response curve method and application to real-time flood forecasting of Fuchunjiang basin. Journal of Hydroelectric Engineering, 2016, 35 ( 1 ) : 38-45. [ 司伟, 余鸿慧, 包为 民等. 面平均雨量的系统响应曲线修正方法及其在富春江流域洪水预报中的应用. 水力发电学报, 2016, 35(1): 38-45.]

[12] Yang Y, Zhang JY, Qi JG et al. Review and prospect on the application of weather radar in hydrology. Advances in Water Science, 2000, (1) : 92-98. [杨扬, 张建云, 戚建国等. 雷达测雨及其在水文中应用的回顾与展望. 水科学进展, 2000 , (1) : 92-98. ]

[13] Collier CG ed. Applications of weather RADAR system. England: Praxis publishing Ltd, 1996.

[14] Artan G, Gadin H, Smith JL et al. Adequacy of satellite-derived rainfall data for streamflow. Natural: Hazards, 2007, 43: 167-185.

[15] Berenguer M, Corral C, Sanchez-Diezma R. Hydrological validation of a radar-based nowcasting technique. Journal of Hydrometeorology, 2005, 6: 532-550.

[16] Si W, Bao W, Wang H et al. The research of rainfall error correction based on system response curve. Applied Mechanics and Materials, 2013, 368-370: 335-339.

[17] Si W, Bao W, Gupta HV. Updating real-time flood forecasts via the dynamic system response curve method. Water Re- 
sources Research, 2015, 51(7) : 5128-5144.

[18] Shu HL, Bao WM, Yang SS et al. Application of runoff error correction based on dynamic system response curve. Water Power, 2016, 42(4) : 22-26. [束慧连, 包为民, 杨姗姗等. 产流误差动态系统响应曲线修正方法的应用. 水力发 电, 2016, $42(4): 22-26$. ]

[19] Sun Y, Bao W, Jiang P et al. Development of multivariable dynamic system response curve method for realtime flood forecasting correction. Water Resources Research, 2018, 54(7) : 4730-4749.

[20] Si W, Gupta HV, Bao W et al. Improved dynamic system response curve method for real-time flood forecast updating. Water Resources Research, 2019.

[21] Si W, Bao WM, Qu SM et al. Real-time flood forecast updating method based on mean areal rainfall error correction. $J$ Lake Sci, 2018, 30(2) : 533-541. DOI: 10.18307/2018.0224. [司伟, 包为民, 篗思敏等. 基于面平均雨量误差修正 的实时洪水预报修正方法. 湖泊科学, 2018, 30(2) : 533-541.]

[22] Bao WM, Chen YT. A conceptual flow-sedimentation coupled simulation model for large basins. Advances in Water Science, 1994, (4) : 287-292. [包为民, 陈耀庭. 中大流域水沙耦合模拟物理概念模型. 水科学进展, 1994, (4): 287-292. ]

[23] Si W, Bao W, Jiang P et al. A semi-physical sediment yield model for estimation of suspended sediment in loess region. International Journal of Sediment Research, 2017, 32(1) : 12-19.

[24] Qu SM, Bao WM. Comprehensive correction of real-time flood forecast. Advances in Water Science, 2003, (2) : 167-171. [翟思敏，包为民. 实时洪水预报综合修正方法初探. 水科学进展, 2003，(2): 167-171.]

[25] Lai SZ, Qu SM, Bao WM et al. Application of parameter calibration method based on parameter function surface in SAC model. Journal of Hydraulic Engineering , 2014, 45(8) : 973-983. [ 赖善证, 睢思敏, 包为民等. 函数曲面参数率定方 法在 SAC 模型中的应用. 水利学报, 2014, 45(8): 973-983.]

[26] Li JY, Zhang N, Wang RB. Soil erosion and sedimentation model in the Yellow River basin: state-of-the-art review. Progress in Geography, 2006, (2) : 103-111. [李景玉, 张楠, 王荣彬. 黄河流域土壤侵蚀产沙模型研究进展. 地理科学 进展, 2006, (2) : 103-111.] 